\title{
Studio di una disequazione quasi-variazionale relativa ad un problema di filtrazione in tre dimensioni $(*)$.
}

\author{
Gtannt GrLaRdi (Pavia) (**)
}

Summary. - A 3-dimensional problem related to the theory of fluid flow through porous media is translated into a quasi-variational inequality: an existence theorem is proved.

\section{Introduzione.}

Questo lavoro tratta di un problema legato allo studio del moto di filtrazione di un fluido attraverso un mezzo poroso in una schematizzazione tridimensionale.

Una diga $D$ di forma generica in materiale poroso, omogeneo e isotropo, sbarra una vallata contenente un liquido omogeneo e incomprimibile formando due bacini, uno "a monte" e uno "a valle" della diga.

Il liquido del bacino a monte passa in quello a valle filtrando attraverso la diga.

In condizioni di moto stazionario e in assenza di fenomeni di capillarità ed evaporazione, il problema consiste nel determinare la parte $\Omega$ della diga $D$ effettivamente attraversata dal liquido e, in ogni punto di $\Omega$, le grandezze fisiche che interessano (ad es. pressione e velocità).

Introdotto un sistema di riferimento $x, y, z$ in modo che l'asse $z$ sia verticale $\theta$ orientato verso l'alto, è noto che, detta $p$ la pressione del liquido (posta uguale a zero la pressione atmosferica e quindi anche la pressione del liquido in $D-\Omega$, se, con opportune scelte delle unità di misura, si considera la quota piezometrica (o potenziale di velocità)

$$
u=p+z \quad \text { in } \Omega
$$

allora si hanno le relazioni (cfr. [9]):

$$
\begin{aligned}
p & =0 \text { in } D-\Omega ; \quad u>z \text { in } \Omega \\
\Delta u & =0 \text { in } \Omega \\
v & =-\operatorname{grad} u \quad \text { in } \Omega
\end{aligned}
$$

$v$ essendo la velocità del liquido.

(*) Entrata in Redazione il 17 marzo 1976.

(**) Lavoro svolto nell'ambito del «Gruppo Nazionale per l'Analisi Funzionale e le sue applicazionị " e dẹl "Laboratorio di Anạlisi Numerica " di Pavia dẹl C.N.R. 
Le condizioni ai limiti si ottengono esprimendo il fatto che lungo le parti permeabili di $\partial D \cap \partial \Omega$ e lungo la "frontiera libera" $D \cap \partial \Omega$ la pressione non ha discontinuità, mentre le parti impermeabile e la frontiera libera non sono attraversate dal liquido.

Estesa la definizione (1) di $u$ a tutto $D$ si ottiene dunque un problema di frontiera libera (ad es. nella incognita $u$ ) che è già stato preso in considerazione da vari autori.

Più precisamente, sono state ampiamente studiate schematizzazioni bidimensionali del problema: in particolare, supponendo verticale la parete a valle della diga e imponendo che la frontiera libera sia un grafico cartesiano decrescente, sono stati dimostrati teoremi di esistenza ed unicità per la soluzione $u$, riconducendo il problema, con opportuno cambiamento di funzione incognita, a un problema di tipo variazionale (cfr. [2], [8] e la bibliografia di [4]; per l'approccio numerico v. ad es. [7]).

Con tecniche analoghe è stato trattato (cfr. [19]) anche il caso tridimensionale nelle seguenti ipotesi : la diga $D$, di base orizzontale ed altezza costante, è delimitata laterlamente da due piani $\Gamma^{\prime}$ e $\Gamma^{\prime \prime}$ verticali e paralleli alla direzione del moto del liquido, e le pareti di monte e di valle della diga sono superficie cilindriche a generatrici verticali, perpendicolari a $\Gamma^{\prime}$ e $\Gamma^{\prime \prime}$ nei punti di intersezione.

Recentemente (v. [5], [12]) ; cfr. anche [3] e [4]) è stato studiato il caso in cui la parete a valle sia inclinata, ma ancora in una schematizzazione bidimensionale, sostituendo la condizione di decrescenza della frontiera libera con una condizione di natura più fisica sulla portata o, equivalentemente, sulla direzione della velocità del liquido nel tratto di "sorgente sospesa", riconducendo il problema iniziale, con opportuno cambiamento di funzione incognita, a un problema di tipo quasi-variazionale e ottenendo teoremi di esistenza per la soluzione.

Nel caso tridimensionale presente si suppone che la vallata, di base orizzontale coincidente col piano $x, y$, sia delimitata lateralmente da superficie cilindriche a generatrici verticali e che la diga $D$ sia invece di forma molto generale.

Introdotto il cambiamento di incognita

$$
U(x, y, z)=\int_{0}^{z} p(x, y, t) d t
$$

è possibile mostrare che il problema iniziale nella incognita $u$ è tradotto nel successivo problema 1.1 in cui l'incognita è appunto $U$ (a tale proposito v. [6]).

In questo lavoro viene preso in esame direttamente il problema 1.1, del quale viene messa in evidenza la natura quasi-variazionale, e viene dimostrato un teorema di esistenza della soluzione minima e della soluzione massima. Il problema dell'unicità della soluzione rimane invece aperto.

Il metodo seguito prende chiaramente spunto da [5], ma con l'introduzione di una variante che, applicata alla situazione bidimensionale, generalizza i risultati di [5]. 


\section{1. - Posizione del problema.}

Una vallata, descritta nello spazio $x, y, z$ dalle disequazioni $Y_{1}(x) \leqslant y \leqslant Y_{2}(x)\left({ }^{1}\right)$, $z \geq 0$, è sbarrata da una diga $D$ delimitata superiormente dalla superficie di equazione $z=Z(x, y)$, la quale taglia la base della vallata secondo due linee $\Sigma_{1}$ e $\Sigma_{2}\left({ }^{2}\right)$ di rispettive equazioni (nel piano $x, y) x=X_{1}(y), x=X_{2}(y)$. Supponiamo che in ciascuno dei due bacini originati dalla diga sia contenuto uno stesso liquido a livelli rispettivi $z_{1}>0, z_{2}>0$.

Denotiamo con $\Gamma^{\prime}$ e $\Gamma^{\prime \prime}$ le superficie della diga a contatto con le pareti della vallata, descritte dunque rispettivamente da $y=Y_{1}(x), 0<z<Z(x, y)$ e $y=Y_{2}(x)$, $0<z<Z(x, y)$; con $\Gamma$ la superficie superiore della diga $D$ e con $\Gamma_{1}$ e $\Gamma_{2}$ le parti di $\Gamma$ "bagnate " dal liquido dei bacini primo e secondo rispettivamente. Denotiamo con $\Gamma_{3}$ la parte "asciutta» di $\Gamma$; con $\Gamma_{0}$ la base di $D$ e poniamo $\Gamma_{N}=\partial D-\Gamma_{0}$.

Denotiamo infine con $B_{i}$ la proiezione ortogonale di $\Gamma_{i}$ sul piano $x, y$ e poniamo $D_{i}=\left\{(x, y, z) \in D:(x, y) \equiv(x, y, 0) \in B_{i} ; 0<z<Z(x, y)\right\} \quad(i=1,2,3)$.

Risulta dunque $D=\left(\bar{D}_{1} \cup \bar{D}_{2} \cup \bar{D}_{3}\right)^{0}, \Gamma_{0}=\left(\bar{B}_{1} \cup \bar{B}_{2} \cup \bar{B}_{3}\right)^{0}, \quad \Gamma=\left(\bar{\Gamma}_{1} \cup \bar{\Gamma}_{2} \cup \bar{\Gamma}_{3}\right)^{0}$, $\Sigma_{i}=\left(\tilde{\Gamma}_{i} \cap \bar{\Gamma}_{0}\right)^{0}(i=1,2)$.

Faremo le seguenti ipotesi:

$\Gamma_{i}$ e $B_{i}$ sono diffeomorfe a un rettangolo $(i=1,2,3)$

$\bar{\Gamma}_{i}$ e $\bar{B}_{i}$ non sono tangenti in alcun punto di $\Sigma_{i}(i=1,2)$

$\bar{\Gamma}$ incontra $\bar{\Gamma}^{\prime}$ e $\bar{\Gamma}^{\prime \prime}$ secondo diedri retti (localmente).

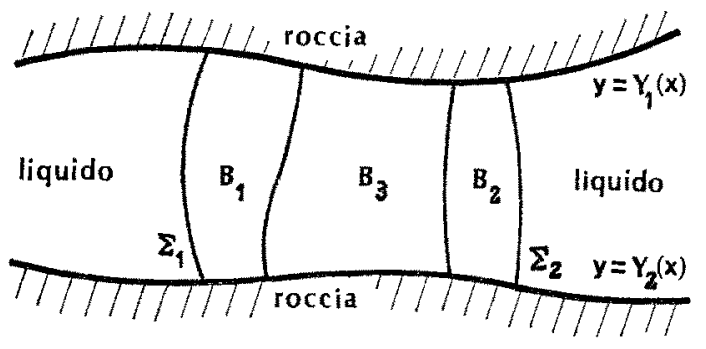

Figura 1

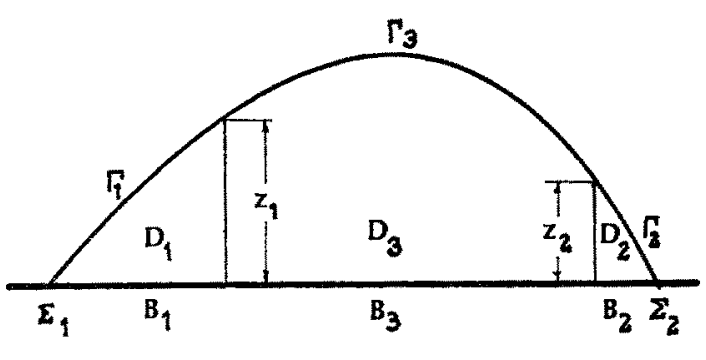

Figura 2

(1) Le funzioni che intervengono nella geometria della diga sono supposte tutte sufficientemente regolari.

(2) $\Sigma_{1}$ e $\Sigma_{2}$ denotano le linee senza estremi; nel seguito le lettere $\Gamma$ e $B$ (anche con apici o indici) denotano varietà bidimensionali senza bordo e $D, D_{i}$ aperti di $\mathbb{R}^{3}$. Le corrispondenti varieta con bordo sono denotate $\bar{\Sigma}_{1}, \bar{\Gamma}, \bar{D}$, ece.

In fig. 1 è descritta la base della diga. In fig. 2 una sezione verticale di $D$ "parallela alla direzione del moto del fluido (asse $y$ ): qui le lettere $T, \Sigma, B, D$ denotano le intersezioni col piano della sezione delle varietà di ugual nome precedentemente definite. 
Supponendo che siano impermeabili la base e le superficie laterali della vallata, che sia poroso, omogeneo e isotropo il materiale di cui è costituita la diga, che il liquido dei bacini sia omogeneo e incomprimibile, che il moto sia in regime stazionario, e trascurando fenomeni di capillarità ed evaporazione, il problema di frontiera libera cui si è accennato nell'introduzione è ricondotto alla risoluzione del problema 1.1 che in questo lavoro ci proponiamo di studiare:

Problema 1.1. - Trovare una funzione $U=U(x, y, z)$ verificante:

$$
\begin{gathered}
U \in H^{2}(D) \cap C^{1}(\bar{D}) \\
\left(\frac{\partial^{2}}{\partial x^{2}}+\frac{\partial^{2}}{\partial y^{2}}\right) U(x, y, Z(x, y)) \geqslant 0 \quad \text { in } B_{3} \\
U_{z}(x, y, z) \geqslant 0 \quad \text { in } \bar{D} \\
\left\{\begin{array}{lll}
U=0 & \text { su } \Gamma_{0} ; \quad U_{z}=0 \quad \text { su } \Gamma_{3} ; \quad U_{z}=z_{i}-z \quad \text { su } \Gamma_{i}(i=1,2) \\
\frac{\partial U}{\partial v}=0 & \text { su } \Gamma^{\prime} \cup \Gamma^{\prime \prime}
\end{array}\right.
\end{gathered}
$$

Posto inoltre

$$
\Omega=D_{1} \cup D_{2} \cup\{(x, y, z) \in D: U(x, y, z)<U(x, y, Z(x, y))\}
$$

risulti:

$$
\Omega \text { contiene un intorno di } \bar{\Gamma}_{\mathbf{0}}\left(^{3}\right)
$$$$
-\Delta U=\chi_{\Omega}\left(^{4}\right) \quad \text { q.o. in } D \text {. }
$$

Osservazione 1.1. - Come si è visto nell'introduzione, $U_{z}$ ha il significato di pressione: è dunque chiara l'interpretazione di (1.4), (1.6), (1.7); inoltre, come si vedrà, $\Omega$ può essere caratterizzato come l'insieme di positività di $U_{z}$; dunque $\Omega$ risulta la parte della diga effettivamente attraversata dal liquido, per cui (1.9) ha ovvio significato; derivando inoltre (1.10) rispetto a $z$ si ritrova l'equazione (3).

Per quanto riguarda la (1.5), si controlla facilmente che essa traduce la condizione che in ogni punto del tratto di sorgente sospesa la velocità non sia diretta verso l'interno della diga. Dal punto di vista fisico sembra sensato richiedere tale condizione

( $\left.{ }^{3}\right)$ La (1.9) va letta: esiste un intorno $W$ di $\bar{\Gamma}_{0}$ in $\mathbb{R}^{3}$ tale che $\Omega \supset W \cap D$.

(4) Con la notazione: $\Delta=\frac{\partial^{2}}{\partial x^{2}}+\frac{\partial^{2}}{\partial y^{2}}+\frac{\partial^{2}}{\partial z^{2}}$. Si utilizzerà anche la notazione $\nabla=$ $\left(\frac{\partial}{\partial x}, \frac{\partial}{\partial y}, \frac{\partial}{\partial z}\right)$. Inoltre $\chi_{A}$ denota la funzione caratteristica dell'inșieme $A$. Si utilizzerà la notazione: $\chi_{i}=\chi_{D_{i}} ; i=1,2,3$, 
solo in casi particolari; già quando il problema si riduca al caso bidimensionale, non ci si aspetta che la condizione sulla direzione della velocità sia soddisfatta se la parete a valle della superficie emersa della diga presenta un certo numero di flessi.

Dunque, sebbene la risoluzione del problema 1.1 non richieda ulteriori proprietà alla funzione $Z$, l'interpretazione fisica sarà corretta solo in determinate condizioni.

\section{2. - Un problema misto lineare.}

1) Si ponga

$$
V=\left\{v \in H^{1}(D):\left.v\right|_{\Gamma_{0}}=0\right\}
$$

munito della norma $\|v\|=\left(\int_{D}\left[v^{2}+|\nabla v|^{2}\right] d D\right)^{\frac{1}{2}}\left(^{5}\right)$. Valgono le relazioni:

$$
\begin{aligned}
& \qquad\left\{\begin{array}{l}
\text { esiste una costante } \beta>0 \text { tale che } \\
\|v\|^{2} \leqslant \beta^{2} \int_{D}|\nabla v|^{2} d D \quad \forall v \in V
\end{array}\right. \\
& \text { l'applicazione }\left.v \rightarrow v\right|_{\Gamma_{N}} \text { è lineare, continua e suriettiva da } V \text { su } H_{00}^{\frac{1}{2}}\left(\Gamma_{N}\right)\left({ }^{6}\right)
\end{aligned}
$$

Si consideri ora la forma bilineare e continua su $V \times V$ :

$$
a(u, v)=\int_{D}\left[\nabla u \cdot \nabla v+Z_{x}\left(u_{x} v_{z}-u_{z} v_{x}\right)+Z_{y}\left(u_{y} v_{z}-u_{z} v_{y}\right)-\left(Z_{x x}+Z_{y y}\right) u_{z} v\right] d D
$$

Posto

$$
M=\max _{\bar{B}}\left|Z_{x x}+Z_{y y}\right| ; \quad \lambda_{0}=2 M^{2}
$$

si ha, per ogni $u \in V$ :

$$
\begin{aligned}
a(u, u)=\int_{D}|\nabla u|^{2} d D-\int_{D}\left(Z_{x x}+Z_{y y}\right) u_{z} u d D \geqslant \int_{D}|\nabla u|^{2} d D-M\left\|u_{z}\right\|_{L^{z}(D)}\|u\|_{L^{z^{2}(D)}} \geqslant & \\
& \geqslant \int_{D}|\nabla u|^{2} d D-\frac{1}{2}\left\|u_{z}\right\|_{L^{2}(D)}^{2}-2 M^{2}\|u\|_{L^{z^{2}(D)}}^{2} .
\end{aligned}
$$

Dunque:

$$
a(u, u)+\lambda\|u\|_{L^{2}(D)}^{2} \geqslant \frac{1}{2 \beta^{2}}\|u\|^{2} \quad \forall u \in V, \forall \lambda \geqslant \lambda_{0}\left({ }^{7}\right) .
$$

(5) $d D$ è l'elemento di volume $d x d y d z$. L'elemento di superficie su $\partial D$ sarà denotato eon $d \sigma$.

$\left(^{6}\right)$ Per la definizione e le proprietà dello spazio $H_{00}^{\frac{1}{2}}$ cfr. [14], 11.

(7) Ovviamente $\lambda=\lambda_{0}$ non è il valore ottimale per la validità di (2.6). 
Per $u$, $v$ regolari si ha inoltre (posto $\nu=\left(v_{x}, \boldsymbol{v}_{y}, \boldsymbol{v}_{z}\right)=$ normale esterna su $\partial D$ ):

$$
\begin{aligned}
a(u, v)=-\int_{D} \Delta u \cdot v d D+\int_{\partial_{D}}\left(v_{x} u_{x}+v_{y} u_{y}+v_{z} u_{z}\right) v d \sigma+ & \\
& +\int_{\partial_{D}}\left[Z_{y} v_{z} u_{y}-Z_{y} v_{y} u_{z}+Z_{x} v_{z} u_{\infty}-Z_{x} v_{\infty} u_{z}\right] v d \sigma .
\end{aligned}
$$

Tenendo conto che si ha $v_{x}+Z_{x} v_{z}=v_{y}+Z_{y} v_{z}=0$ e $v_{z}=1 / \sqrt{1+Z_{x}^{2}+Z_{y}^{2}}$ su $T$, $v_{z}=Z_{x} v_{x}+Z_{y} v_{y}=0$ su $\Gamma^{\prime} \cup \Gamma^{\prime \prime}$ (cfr. la (1.3)), se $u$, $v$ sono nulle su $\Gamma_{0}$, si ha:

$$
a(u, v)=-\int_{D} \Delta u \cdot v d D+\int_{\Gamma} \sqrt{1+Z_{x}^{2}+Z_{y}^{2}} \cdot u_{z} v d \sigma+\int_{\Gamma^{\prime}} \frac{\partial u}{\partial v} v d \sigma .
$$

Posto, per $u$ regolare:

$$
\gamma_{1} u= \begin{cases}\sqrt{1+Z_{x}^{2}+Z_{y}^{2} \cdot u_{z}} & \text { su } \Gamma \\ \frac{\partial u}{\partial v} & \text { su } \Gamma^{\prime} \cup \Gamma^{\prime \prime}\end{cases}
$$

e considerato lo spazio $H_{\Delta}^{1}(D)=\left\{v \in H^{1}(D): \Delta v \in L^{2}(D)\right\}$ munito della norma del grafico, l'operatore $\gamma_{1}$ si prolunga per densità in un operatore, denotato ancora con $\gamma_{1}$, lineare e continuo da $H_{\Delta}^{1 *}(D)$ in $\left(H_{00}^{\frac{1}{2}}\left(\Gamma_{N}\right)\right)^{\prime}$ in modo che valga la formula di Green:

$$
a(u, v)=-\int_{D} \Delta u \cdot v d D+{ }_{\left(H_{00}^{\frac{1}{2}}\left(\Gamma_{N}\right)\right)^{\prime}}\left\langle\gamma_{1} u,\left.v\right|_{\Gamma_{N}}\right\rangle_{\left(B_{00}^{\frac{1}{t}}\left(\Gamma_{N}\right)\right)} \quad \forall u \in H_{A}^{1}(D), \forall v \in V .
$$

Definita ora la funzione su $\Gamma$ :

$$
g(x, y, z)= \begin{cases}z_{i}-z & \text { su } \Gamma_{i} ; i=1,2 \\ 0 & \text { su } \Gamma_{3} .\end{cases}
$$

Saremo interessati allo studio del problema seguente:

fissati $\lambda$ reale e $h \in L^{2}(D)$, trovare $u$ verificante:

$$
\left\{\begin{array}{l}
u \in V, \\
a(u, v)+\lambda \int_{D} u v d D=\int_{D} h v d D+\int_{\Gamma} \sqrt{1+Z_{\infty}^{2}+Z_{y}^{2}} \cdot g v d \sigma, \quad \forall v \in V .
\end{array}\right.
$$

Una tale funzione $u$ verifica

$$
\begin{cases}-\Delta u+\lambda u=h & \text { in } \mathcal{D}^{\prime}(D), \\ \left.u\right|_{\Gamma_{0}}=0 & \text { in } H^{\frac{1}{2}}\left(\Gamma_{0}\right), \\ u_{z \mid \Gamma}=g & \text { in }\left(H_{00}^{\frac{1}{2}}(\Gamma)\right)^{\prime}, \\ \left.\frac{\partial u}{\partial \nu}\right|_{\Gamma^{\prime} \cup \Gamma^{\prime}}=0 & \text { in }\left(H_{00}^{\frac{3}{2}}\left(\Gamma^{\prime} \cup \Gamma^{\prime \prime}\right)\right)^{\prime} .\end{cases}
$$


I risultati di [15], [16] e [11] forniscono i teoremi seguenti:

TeORema 2.1. - Con le notazioni (2.5), (2.7), fissati comunque $\lambda \geqslant \lambda_{0}$ e $h \in L^{2}(D)$, esiste una ed una sola u verificante (2.8). Esistono inoltre $\alpha \in] 0,1[$ e $C>0$ (indipen. denti da he da u) tali che:

$$
\left\{\begin{array}{l}
u \in O^{0, \alpha}(\bar{D}) \\
\|u\|_{B^{1}(D)}+\|u\|_{0^{0, \alpha}(\bar{D})} \leqslant C\left[\|h\|_{L^{2}(D)}+1\right]
\end{array}\right.
$$

Teorema 2.2. - Se $\lambda=0$, fissata comunque $h \in L^{2}(D)$, il problema (2.8) ha una ed una sola soluzione $u$.

2) Ci proponiamo di studiare la regolarità delle eventuali soluzioni del problema (2.8) nel caso particolare in cui $h$ sia della forma:

$$
h=\lambda w+h^{0} ; w \in V \text { e hölderiana in } \bar{D} ; h^{0} \in L^{6}(D) ; h^{0} \equiv 1 \text { in } D_{1} \cup D_{2}\left(^{8}\right)
$$

Sussiste il seguente

TEовемa 2.3. - Fissato comunque $\lambda$ reale, se g e $h$ verificano (2.8) e (2.11), allora ogni soluzione u del problema (2.9) appartiene allo spazio $W^{2,6}(D)$.

Risulta in particolare $u \in O^{1}(\bar{D})$.

DiM. - Procediamo in varie tappe. Sia $u \in H^{1}(D)$ una soluzione di (2.9).

Passo 1. Essendo $H^{1}(D) \subset L^{6}(D)$, (2.11) fornisce $h \in L^{6}(D)$. Grazie ai risultati di [1], $u$ è allora di classe $W^{2,6}$ nell'intorno di ogni punto appartenente a $D$ o alle parti regolari di $\partial D$. Inoltre, $u$ è ancora di classe $W^{2,6}$ nell'intorno dei punti di $\partial \Gamma^{\prime} \cup \partial \Gamma^{\prime \prime}$ che non siano estremi degli spigoli $\bar{\Sigma}_{1}$ e $\bar{\Sigma}_{2}$, in quanto con opportuno ribaltamento ci si riconduce grazie a (1.3) ad una situazione analoga alla precedente. Quindi

$$
\left\{\begin{array}{l}
\text { fissato comunque un aperto } D_{0} \subset D \text { tale che } \bar{D}_{0} \subset \bar{D}-\left(\bar{\Sigma}_{1} \cup \bar{\Sigma}_{2}\right) \\
\text { risulta }\left.u\right|_{D_{0}} \in W^{2,6}\left(D_{0}\right)
\end{array}\right.
$$

Passo 2. Mostriamo che $u_{z}$ è di classe $H^{2}$ nell'intorno dei punti di $\bar{\Sigma}_{\mathbf{1}} \cup \bar{\Sigma}_{2}$. Basta ragionare relativamente a $\bar{\Sigma}_{1}$.

Supponiamo dapprima $P_{0} \in \Sigma_{1}$. Imitando [5] poniamo: $v(x, y, z)=u_{z}(x, y, z)+$ $+z-z_{1}$. Si ha $v \in L^{2}\left(D_{1}\right)$ e $\left.v\right|_{\Gamma_{1}}=0$.

Inoltre: $\Delta u=\Delta u_{z}=(\Delta u)_{z}=\lambda\left(u_{z}-w_{z}\right)$ in $D_{1}$, grazie a (2.11); dunque $\Delta v \in L^{2}\left(D_{1}\right)$. Ma, vicino a $\Gamma_{0},\left.u\right|_{D_{1}}$ è regolare essendo $\Delta u$ hölderiano in $\bar{D}_{1}$; quindi è possibile calcolare $v_{x}(x, y, 0)$ vicino a $P_{0}$ con regole classiche.

${ }^{8}$ ) Ovviamente (2.11) può essere espressa in forma notevolmente più semplice. 
Si ha su $B_{1}: v_{z}=u_{z z}+1=\Delta u-u_{q x}-u_{y y}+1=\Delta u+1=\lambda(u-w)-h^{0}+1=0$, grazie a (2.11). Introducendo gli insiemi $\breve{D}_{1}=\left\{(x, y, z):(x, y) \in B_{1} ;|z|<Z(x, y)\right\}$, $\breve{\Gamma}_{1}=\left\{(x, y, z) ;(x, y) \in B_{1} \cup \Sigma_{1} ;|z|=Z(x, y)\right\}$ e la funzione $\check{v}(x, y, z)=v(x, y,|z|)$, si ha allora: $\breve{v} \in L^{2}\left(\breve{D}_{1}\right), \Delta \breve{v} \in L^{2}\left(\breve{D}_{1}\right),\left.\breve{v}\right|_{T_{1}-\Sigma_{1}}=0$. Ma in tali condizioni si ha $\left.v\right|_{\Gamma^{1}} \in$ $\in\left(H_{00}^{\frac{1}{2}}\left(\breve{\Gamma}_{1}\right)\right)^{\prime} ;\left(^{9}\right)$ dunque deve essere $\left.\breve{v}\right|_{\check{\Gamma}_{1}} \equiv 0$.

Quindi $\left(^{\left({ }^{10}\right)} \breve{v} \in H^{2}\right.$ di un intorno di $P_{0}$, da cui si deduce che $u_{z}$ è di classe $H^{2}$ vicino a $P_{0}$.

Se ora $P_{0} \in \partial \Sigma_{1}$, con opportuno ribaltamento, si è ricondotti ad una situazione analoga alla precedente.

Passo 3. Proviamo che $u \in H^{2}(D)$.

Grazie a (2.12), basta ragionare vicino a $\bar{\Sigma}_{1} \cup \bar{\Sigma}_{2}$. Ancora, sarà sufficiente mostrare la regolarità $H^{2}$ vicino ai punti di $\Sigma_{1}$. Sia dunque $P_{0} \equiv\left(x_{0}, y_{0}, 0\right) \in \Sigma_{1}$; scelto $\varepsilon>0$ minore della distanza di $P_{0}$ da $\partial B_{1}-\Sigma_{1}$, si scelga $\varphi$ verificante:

$$
\varphi \in C^{\infty}[0, \infty[; \quad \varphi \equiv 1 \text { in }[0, \varepsilon / 3] ; \quad \varphi \equiv 0 \text { in }[2 \varepsilon / 3, \infty[
$$

e si ponga:

$$
v(x, y, z)=u(x, y, z) \cdot \varphi\left(\sqrt{\left.\left(x-x_{0}\right)^{2}+\left(y-y_{0}\right)^{2}\right)} \quad \text { in } D .\right.
$$

Posto $D_{\sigma}=\left\{(x, y, z) \in D:\left(x-x_{0}\right)^{2}+\left(y-y_{0}\right)^{2}<\sigma^{2}\right\}, \sigma>0$, si ha:

$$
\left\{\begin{array}{l}
v \in H^{1}\left(D_{\varepsilon}\right) \\
\Delta v \in L^{2}\left(D_{\varepsilon}\right) \\
v_{x \mid \Gamma \cap \partial D_{\varepsilon}}=\text { funzione regolare } \\
\left.v\right|_{\Gamma_{0} \cap \partial D_{\varepsilon}}=0 \\
\operatorname{supp} v \subset D_{2 \varepsilon / 3} .
\end{array}\right.
$$

Si introduca la trasformazione $T: \bar{D}_{\varepsilon} \rightarrow \mathbf{R}^{s}$ definita da $T(x, y, z)=(\xi, \eta, \zeta)$ ove:

$$
\left\{\begin{array}{l}
\xi=x-X_{1}(y) \\
\eta=y-y_{0} \\
\zeta=\frac{z}{Z(x, y)}\left(x-X_{1}(y)\right) .
\end{array}\right.
$$

Grazie a (1.2) e alla regolarità di $X_{1}$ e $Z, T$ risulta un diffeomorfismo di $D_{\varepsilon}$ sull'aperto $T\left(D_{\varepsilon}\right)$ del diedro $\widetilde{D}=\{(\xi, \eta, \zeta): 0<\zeta<\xi\}$ e inoltre $T\left(P_{0}\right)=(0,0,0)$.

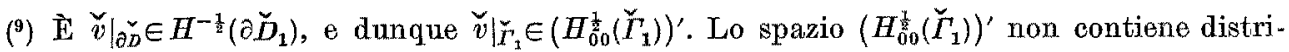
buzioni con supporto contenuto in una varietà di dimensione 1 .

(10) Per (1.2) $\check{D}_{\mathfrak{1}}$ è diffeomorfo, nell'intorno dí $P_{0}$, a un diedro convesso di $\mathbb{R}^{3}$. 
Considerata la funzione $\tilde{v}: \tilde{D} \rightarrow \mathbb{R}$ definita da: $\tilde{v}(\xi, \eta, \zeta)=v\left(T^{-1}(\xi, \eta, \zeta)\right)$ in $T\left(D_{\varepsilon}\right)$, $\tilde{v}(\xi, \eta, \zeta)=0$ in $\widetilde{D}-T\left(D_{\varepsilon}\right)$, da $(2.15)$ si deduce, con $\mu$ reale qualunque:

$$
\begin{cases}\tilde{v} \in H^{1}(\tilde{D}) & \\ \mathfrak{A} \tilde{v}+\mu \tilde{v}=F_{\mu} & \text { in } \tilde{D} \\ \mathfrak{B} \tilde{v}=G & \text { per } \zeta=\xi \\ \tilde{v}=0 & \text { per } \zeta=0\end{cases}
$$

ove $\mathcal{A}$ è un operatore uniformemente ellittico su $\tilde{D}, \mathcal{B}$ un operatore di derivata obliqua regolare, $F_{\mu}$ una funzione di $L^{2}(\tilde{D})$ e $G$ una funzione regolare (e con supporto limitato) sulla faccia $\zeta=\xi$ di $\widetilde{D}$.

Ma, per $\mu$ abbastanza grande, il problema (2.17) è variazionale e il dominio $\tilde{D}$ e l'ultima delle (2.17) (cioè la condizione forzata) sono invarianti rispetto alle traslazioni nella direzione dell'asse $\eta$. Dunque, passando al limite sui rapporti incrementali, si deduce:

$$
\frac{\partial \tilde{v}}{\partial \eta} \in H^{x}(\tilde{D})
$$

Esprimendo (2.18) mediante le variabili $x, y, z$ ed essendo ormai noto che $v_{z} \in H^{1}(D)$, si deduce immediatamente che $v_{x} \cdot X_{1}^{\prime}(y)+v_{y} \in H^{1}\left(D_{\varepsilon}\right)$, da cui

$$
v_{x x} \cdot X_{1}^{\prime}(y)+v_{x y} \in L^{2}\left(D_{\varepsilon}\right) ; \quad v_{x y} \cdot X_{1}^{\prime}(y)+v_{y y} \in L^{2}\left(D_{\varepsilon}\right)
$$

Ma, da $\Delta v \in L^{2}\left(D_{\varepsilon}\right)$ e da $v_{z} \in H^{1}(D)$ si deduce

$$
v_{x: x}+v_{y y} \in L^{2}\left(D_{\varepsilon}\right) .
$$

Allora (2.19) e (2.20) forniscono $v_{x x}, v_{x y}, v_{y y} \in L^{2}\left(D_{\varepsilon}\right)$ che, unite a $v_{x} \in H^{1}(D)$, assicurano che $v \in H^{2}\left(D_{\varepsilon}\right)$. Ne segue $u \in H^{2}\left(D_{\varepsilon / 3}\right)$.

Passo 4. Proviamo che $u \in W^{2,6}(D)$.

Ancora basterà fissare $P_{0} \equiv\left(x_{0}, y_{0}, 0\right) \in \Sigma_{1}$ e provare che $u$ è di classe $W^{2,6}$ vicino a $P_{0}$. Procedendo come al passo 3 , ne riutilizziamo le notazioni. Ora è noto che $v \in H^{2}(D)$ e quindi che $v_{x}, v_{y} \in H^{x}(D)$; inoltre il calcolo esplicito di $\Delta v$ mostra ora che $\Delta v \in H^{1}\left(D_{z}\right)$, da eui $\Delta v_{x}, \Delta v_{y} \in L^{2}\left(D_{\varepsilon}\right)$, mentre $\left(v_{x}\right)_{z}$ e $\left(v_{y}\right)_{z}$ sono funzioni regolari su $\Gamma \cap \partial D_{\varepsilon}$ e $v_{x}=v_{y}=0$ su $\Gamma_{0} \cap \partial D_{\varepsilon}$.

Inoltre i supporti di $v_{x}$ e $v_{z}$ sono contenuti in $\bar{D}_{2 \varepsilon / 3}$.

Dunque $v_{x}$ e $v_{y}$ verificano relazioni analoghe alla (2.15) e quindi, con la notazione (2.16), si ottengono per $v_{x}$ e $v_{y}$ le analoghe della (2.18).

Tenendo ora conto del fatto che $H^{1}\left(D_{\varepsilon}\right) \subset L^{6}\left(D_{\varepsilon}\right)$, dalle relazioni trovate e dal fatto che $v_{z} \in H^{2}(D)$ si deducono le analoghe di $(2.19),(2.20)$ con esponente di sommabilità 6 anzichè 2 , da cui si ottengono le relazioni $v_{x x}, v_{x y}, v_{y y} \in L^{6}\left(D_{\varepsilon}\right)$ che, unite a $v_{z} \in W^{1,6}\left(D_{\varepsilon}\right)$, assicurano che $v \in W^{2,6}\left(D_{\varepsilon}\right)$. Ne segue $u \in W^{2,6}\left(D_{\varepsilon / 3}\right)$. c.v.d. 


\section{3. - Un problema non lineare.}

1) Con la notazione (2.7), consideriamo il problema: fissato $\lambda$ reale e assegnate $f, w$ verificanti

$$
f \in C_{0}\left(\bar{B}_{3}\right)\left({ }^{11}\right) ; \quad w \in W^{2,6}(D) \cap V
$$

trovare $u$ verificante:

$$
\left\{\begin{array}{l}
u \in V, \\
a(u, u-v)+\lambda \int_{D} u(u-v) d D+\int_{D_{s}}[f-u]+d D \leqslant \lambda \int_{D} w(u-v) d D+ \\
+\int_{D_{3}}[f-v]+d D+\int_{D_{1} \cup D_{2}}(u-v) d D+\int_{\Gamma} \sqrt{1+Z_{x}^{2}+Z_{y}^{2}} \cdot g(u-v) d \sigma, \quad \forall v \in V .
\end{array}\right.
$$

Imitando [5̆] e tenendo conto del teor. 2.3 si controlla $\left({ }^{12}\right)$ la validità del seguente:

TEOREMA 3.1. - Fissati comunque $\lambda$ reale $e t$, w verificanti (3.1), ogni soluzione $u$ di (3.2) verifica:

$$
\begin{array}{ll}
u \in W^{2,6}(D) & \\
0 \leqslant-\Delta u+\lambda(u-w) \leqslant 1 & \text { in } D \\
-\Delta u+\lambda u=\lambda w+1 \quad \text { in } D_{1} \cup D_{2} \\
\left.u\right|_{\Gamma_{0}}=0 ; \quad u_{z \mid \Gamma^{\prime}}=g ;\left.\quad \frac{\partial u}{\partial y}\right|_{\Gamma^{\prime} \cup \Gamma^{\prime}}=0 .
\end{array}
$$

Posto inoltre $\Omega^{=}=\left\{(x, y, z) \in D_{3}: u(x, y, z) \lessgtr f(x, y)\right\}$, risulta:

$$
-\Delta u+\lambda u=\lambda w+1 \text { in } \Omega^{+} ; \quad-\Delta u+\lambda u=\lambda w \text { in } \Omega^{-} \text {. }
$$

Riuniremo (3.4), (3.5), (3.7) nella relazione

$$
-\Delta u+\lambda u \in \lambda w+\chi_{1}+\chi_{2}+\chi_{3} H(f-u)
$$

ove $H$ è il grafo massimale monotono definito da: $H(t)=\{0\}$ per $t<0, H(0)=[0,1]$; $H(t)=\{1\}$ per $t>0$.

(11) Si penserà di denotare ancora con $f$ anche la funzione definita in $D_{3}:(x, y, z) \rightarrow f(x, y)$.

$\left({ }^{12}\right)$ Si controllano dapprima $(3.4),(3.5),(3.6)$ in senso generalizzato. Il teor. 2.3 fornisce allora $(3,3)$. In particolare $Q^{ \pm}$sono ben definiti e aperti. 
I risultati di [1.5] e la (2.6) forniseono:

TeORema 3.2. - Con la notazione (2.5) e nelle ipotesi (3.1), fissato comunque $\lambda \geqslant \lambda_{0}$ esiste una ed una sola u verificante (3.2).

Consideriamo ora il problema: in $\left.B_{3}\right\}$.

fissata $\psi \in W^{1, p}\left(B_{3}\right)$ con $p>2$, si ponga $K_{\psi}=\left\{v \in H^{1}\left(B_{3}\right): v-\psi \in H_{0}^{1}\left(B_{3}\right) ; v \leqslant \psi\right.$

Il problema consiste nel trovare $f$ verificante:

$$
\left\{\begin{array}{l}
f \in K_{w} \\
\int_{B_{\mathbf{s}}}\left[f_{x}\left(f_{x}-v_{x}\right)+f_{y}\left(f_{y}-v_{y}\right)\right] d x d y \leqslant 0, \quad \forall v \in K_{p} .
\end{array}\right.
$$

I risultati di [16] assicurano che esiste una ed una sola $f$ verificante (3.9) e che tale $f$ risulta hölderiana in $\bar{B}_{3}$.

Poniamo:

$$
f=\$ \psi
$$

È di verifica immediata (13) il fatto seguente:

$$
\left\{\begin{array}{l}
\text { l'operatore } S \text { è monotono non decrescente e continuo rispetio alla topologia } \\
d i C^{0}\left(\bar{B}_{3}\right) .
\end{array}\right.
$$

Introdotta ora la notazione

$$
\left(\gamma_{3} u\right)=u(x, y, Z(x, y)) ; \quad \forall(x, y) \in \bar{B}_{3}, \forall u \in C^{0}\left(\bar{D}_{3}\right)
$$

si osserva che se $w \in W^{2,6}(D)$, allora la funzione $\delta \gamma_{3} w$ è sicuramente definita.

In particolare, per i risultati precedenti, si ha:

Teorema 3.3. - Con le notazioni (2.5), (2.7), fissata comunque $w \in W^{2,6}(D) \cap V$, esiste una ed una sola soluzione u del problema:

$$
\left\{\begin{array}{l}
u \in W^{2,6}(D) ; \quad \text { valgano } l e(3.6) \\
-\Delta u+\lambda_{0} u \in \lambda_{0} w+\chi_{1}+\chi_{2}+\chi_{3} H\left(\delta \gamma_{3} w-u\right) .
\end{array}\right.
$$

Introduciamo allora la notazione:

$$
u=\mathfrak{W}\left(^{(14}\right)
$$

(13) Per il principio di massimo. A questo proposito $\nabla$. [11] e [17].

$\left({ }^{14}\right)$ Perchè $\bar{D} w$ sia definita, ovviamente non occorrono ipotesi su $w$ tanto restrittive. 
2) Ci proponiamo ora di studiare alcune proprietà dell'operatore $\mathfrak{C}$.

Lemar 3.1. - Se $w_{1}, w_{2} \in W^{2,6}(D) \cap V$ e se $w_{1} \leqslant w_{2}$ in D, allora $\mathcal{C} w_{1} \leqslant \mathscr{C} w_{2}$.

Dmr. - Pongo $u_{i}=\mathcal{G}_{w_{i}}$ e considero l'insieme aperto $\omega=\{(x, y z) \in D$ : $u_{1}(x, y, z)>u_{2}(x, y,(z)$, supponendo per assurdo $\omega \neq \emptyset$.

Grazie a (2.11), in $\omega$ si ha $\delta \gamma_{3} w_{1}-u_{1}<\delta \gamma_{3} w_{2}-u_{2}$ da cui $-\Delta u_{1}+\lambda_{0}\left(u_{1}-w_{1}\right) \leqslant$ $\leqslant-\Delta u_{2}+\lambda_{0}\left(u_{2}-w_{2}\right)$ e quindi $-\Delta u_{1} \leqslant-\Delta u_{2}$ in $\omega$. Per il principio di massimo si ha allora $u_{1} \leqslant u_{2}$ in $\omega$, che contraddice la definizione di $\omega$. c.v.d.

Introduciamo le notazioni:

$$
\left\{\begin{array}{l}
\text { oon } u_{0} \text { e } u^{0} \text { denotiamo le funzioni } u \text { date dal teor. } 2.2 \text { con } h=\chi_{1}+\chi_{2} \\
e h \equiv 1 \text { rispettivamente. }
\end{array}\right.
$$

È di dimostrazione immediata il seguente $\left({ }^{15}\right)$ :

LEMMA 3.2. - Risulta

$$
0 \leqslant u_{0} \leqslant u^{0} \quad \text { in } \bar{D} .
$$

Inoltre per ogni $w \in W^{2,6}(D) \cap V$ si ha:

$$
\text { se } u_{0} \leqslant w \leqslant u^{0} \text { in } \bar{D} \text { allora } u_{0} \leqslant \mathscr{G} w \leqslant u^{0} \text { in } \bar{D} \text {. }
$$

Definizione 3.1. - Fissata $w \in W^{2,6}(D) \cap V$, con le notazioni (2.5), (2.7), la soluzione $u$ del problema (2.8) con $\lambda=\lambda_{0}$ e $h=\lambda_{0} w+1$ verrà denotata con $\mathfrak{C}^{*} w$.

LeMma 3.3. - Se $w \in W^{2,6}(D) \cap V$ si ha $\mathfrak{C}^{*} w \in W^{2,6}(D)$ e $\gamma_{3} \mathcal{G}^{*} w \in C^{2}\left(B_{3}\right)$.

Dim. - Il teor. 2.3 fornisce $\mathfrak{C}^{*} w \in W^{2, \theta}(D)$. Per ottenere l'altra tesi basta osservare che $\mathfrak{C}^{*} w \in C^{2}\left(D_{3} \cup \Gamma_{3}\right)$ essendo $\Delta \mathcal{G}^{*} w \in C^{1}(\bar{D})$. c.v.d.

Si introduca ora, per $m$ intero $>0$ fissato, la funzione $H_{m}: \mathbb{R} \rightarrow \mathbb{R}$ definita da: $H_{m}(t)=0$ per $t \leqslant-2 / m ; H_{m}(t)=m t+2$ per $-2 / m \leqslant t \leqslant-1 / m ; H_{m}(t)=1$ per $t \geqslant-1 / m$.

Si controlla facilmente che, con le notazioni (2.5), (2.7), fissata $w \in W^{2,6}(D) \cap V$, esiste una ed una sola $u$ verificante

$$
\left\{\begin{array}{l}
u \in H^{1}(D) \\
-\Delta u+\lambda_{0} u=\lambda_{0} w+\chi_{1}+\chi_{2}+\chi_{3} \cdot H_{m}\left(\S \gamma_{3} w-u\right) \quad \text { in } \mathcal{D}^{\prime}(D) \\
\left.u\right|_{\Gamma_{0}}=0 ; \quad u_{z \mid \Gamma}=g ;\left.\quad \frac{\partial u}{\partial v}\right|_{\Gamma^{\prime} \cup \Gamma^{\prime \prime}}=0 \quad \text { nel senso dei teoremi di tracce. }
\end{array}\right.
$$

Denotata tale funzione $u$ con $\mathfrak{G}^{m} w$, si ha inoltre $\lim _{m \rightarrow \infty} \mathfrak{C}^{m} w=\mathfrak{G} w$, ad es. in $\Pi^{1}(D)$.

(15) Si applicano i principi di massimo e di Hopf. Per escludere che punti di massimo e di minimo siano su $\bar{\Gamma}^{\prime}>\bar{\Gamma}^{\prime \prime}$, si procede con opportuno ribaltamento. 
Lemra 3.4. - Se $w \in W^{2,6}(D) \cap V$ verifica $w_{z} \geqslant 0$ in $\bar{D}$ allora per ogni $m>0$ si $h a: \mathfrak{G}^{m} w \geqslant 0,(\partial / \partial z) \mathfrak{C}^{m} w \geqslant 0$ in $D$.

Drm. - Posto $u^{m}=\mathfrak{C}^{m} w$, si osservi che $u^{m} \in W^{2,6}(D)$, grazie al teor. 2.3.

Tenendo conto che dall'ipotesi segue $w \geqslant 0$ in $\bar{D}$, la prima tesi è conseguenza dei principi di massimo e di Hopf. Segue in particolare: $u_{z}^{m} \geqslant 0 \mathrm{su} \Gamma_{0}$. Per dimostrare la seconda disuguaglianza si supponga non vuoto l'aperto $\omega=\left\{(x, y, z) \in D: u_{z}^{m}(x, y, z)<0\right\}$. In $\omega$ si ha $A u_{z}^{m}=\lambda_{0}\left(u_{z}^{m}-w_{z}\right)+\chi_{3} \cdot H_{m}^{\prime}\left(\delta \gamma_{3} w-u^{m}\right) \cdot u_{z}^{m} \leqslant 0$. Dunque $u_{z}^{m} \geqslant 0$ in $\bar{\omega}$, che contraddice la definizione di $\omega$. e.v.d.

Lemma $3.5 .-$ Se $w \in W^{z, 6}(D) \cap V$ verifica $w_{z} \geqslant 0$ in $\bar{D}$ allora $\mathcal{G}^{m} w \leqslant \mathcal{G}^{*} w$, $(\partial / \partial z) \mathcal{C}^{m} w \leqslant(\partial / \partial z) \mathfrak{C}^{*} w$ in $\bar{D}$.

DrM. - Posto $u^{m}=\mathfrak{C}^{m} w, u^{*}=\mathfrak{C}^{*} w$, supponiamo non vuoto l'aperto $\omega=$ $=\left\{(x, y, z) \in D: u^{m}(x, y, z)>u^{*}(x, y, z)\right\}$. In $\omega$ si ha: $\Delta\left(u^{m}-u^{*}\right)=\lambda_{0}\left(u^{m}-u^{*}\right)-\chi_{1}-$ $-\chi_{2}-\chi_{3} \cdot H_{m}\left(\delta \gamma_{3} w-u^{m}\right)+1 \geqslant \lambda_{0}\left(u^{m}-u^{*}\right)>0$. Dunque $u^{m} \leqslant u^{*}$ in $\bar{\omega}$ che è assurdo. Quindi $u^{m} \leqslant u^{*}$ in $\widetilde{D}$, che è la prima tesi.

Osservato che, in particolare, si ha $u_{z}^{m} \leqslant u_{z}^{*}$ su $\Gamma_{0}$, si supponga ora non vuoto l'aperto $\omega^{\prime}=\left\{(x, y, z) \in D: u_{z}^{m}(x, y, z)>u_{z}^{*}(x, y, z)\right\}$. In $\omega^{\prime}$ si ha: $\Delta\left(u_{z}^{m}-u_{z}^{*}\right)=$ $=\lambda_{0}\left(u_{z}^{m}-u_{z}^{*}\right)+\chi_{3} \cdot H_{m}^{\prime}\left(\delta \gamma_{3} w-u^{m}\right) \cdot u_{z}^{m} \geqslant 0$, per il lemma 3.4 .

Dunque $u_{z}^{m} \leqslant u_{z}^{*}$ in $\bar{\omega}^{\prime}$, da cui la seconda tesi.

LEMMA 3.6. - Se $w \in W^{2,6}(D) \cap V$ verifica $w_{z} \geqslant 0$ in $\bar{D}$ allora si ha

$$
\left(\frac{\partial^{2}}{\partial x^{2}}+\frac{\partial^{2}}{\partial y^{2}}\right)\left(\gamma_{3} \mathcal{C}^{m} w\right) \leqslant\left(\frac{\partial^{2}}{\partial x^{2}}+\frac{\partial^{2}}{\partial y^{2}}\right)\left(\gamma_{3} \mathcal{C}^{*} w\right)+1 \quad \text { in } B_{3}
$$

DLy. - Posto $u^{m}=\mathfrak{C}^{m} w, u^{*}=\mathfrak{C}^{*} w, u^{m}$ e $u^{*}$ sono di classe $C^{2}$ vicino a $\Gamma_{3}$. Essendo $\left(u^{m}-u\right)_{z}(x, y, Z(x, y)) \equiv 0$ in $B_{3}$, si ha in $B_{3}$ :

$$
\begin{aligned}
& \left(u^{\eta_{n}}-u^{*}\right)_{x z}(x, y, Z(x, y))+Z_{x}(x, y) \cdot\left(u^{m}-u^{*}\right)_{z z}(x, y, Z(x, y))=0 \\
& \left(u^{m_{z}}-u^{*}\right)_{y z}(x, y, Z(x, y))+Z_{y}(x, y) \cdot\left(u^{m}-u^{*}\right)_{z z}(x, y, Z(x, y))=0 .
\end{aligned}
$$

Tenendo conto che dal lemma 3.5 si deduce $\left(u^{m_{0}}-u^{*}\right)_{z z}(x, y, Z(x, y)) \geqslant 0$, si ha allora in $B_{3}$ :

$$
\begin{aligned}
& \left(\frac{\partial^{2}}{\partial x^{2}}+\frac{\partial^{2}}{\partial y^{2}}\right)\left[\left(u^{m}-u^{*}\right)(x, y, Z(x, y))\right]= \\
& \quad=\frac{\partial}{\partial x}\left[\left(u^{m}-u^{*}\right)_{x}(x, y, Z(x, y))+Z_{x}(x, y) \cdot\left(u^{m}-u^{*}\right)_{z}(x, y, Z(x, y))\right]+ \\
& \quad+\frac{\partial}{\partial y}\left[\left(u^{m}-u^{*}\right)_{y}(x, y, Z(x, y))+Z_{y}(x, y) \cdot\left(u^{m}-u^{*}\right)_{z}(x, y, Z(x, y))\right]= \\
& \quad=\left(u^{m}-u^{*}\right)_{x x}(x, y, Z(x, y))+Z_{x}(x, y) \cdot\left(u^{m}-u^{*}\right)_{x z}(x, y, Z(x, y))+ \\
& \quad 2-\text { Annali di Matematica }
\end{aligned}
$$




$$
\begin{aligned}
& +\left(u^{m}-u^{*}\right)_{y y}(x, y, Z(x, y))+Z_{y}(x, y) \cdot\left(u^{m}-u^{*}\right)_{y z}(x, y, Z(x, y))= \\
& =\left[\Delta\left(u^{m}-u^{*}\right)\right](x, y, Z(x, y))-\left[1+Z_{x}^{2}(x, y)+Z_{y}^{2}(x, y)\right] \cdot\left(u^{m}-u^{*}\right)_{z x}(x, y, Z(x, y)) \leqslant \\
& \leqslant\left[\Delta\left(u^{m}-u^{*}\right)\right](x, y, Z(x, y))= \\
& \quad \lambda_{0}\left[u^{m}(x, y, Z(x, y))-u^{*}(x, y, Z(x, y))\right]+ \\
& +1-\left[H_{m}\left(\delta \gamma_{3} w-u^{m}\right)\right](x, y, Z(x, y)) \leqslant 1 . \quad \text { c.v.d. }
\end{aligned}
$$

Segue immediatamente il:

COROLIaRIO 3.1. - Se $w \in W^{2,6}(D) \cap V$ verifica $w_{z} \geqslant 0$ in $\bar{D}$ allora

$$
\left(\frac{\partial^{2}}{\partial x^{2}}+\frac{\partial^{2}}{\partial y^{2}}\right)\left(\gamma_{3} \mathscr{C} w\right) \leqslant\left(\frac{\partial^{2}}{\partial x^{2}}+\frac{\partial^{2}}{\partial y^{2}}\right)\left(\gamma_{3} \mathscr{C}^{*} w\right)+1 \quad \text { in } \mathfrak{D}^{\prime}\left(B_{3}\right)
$$

\section{4. - Riconduzione del problema 1.1 ad un problema quasi-variazionale.}

1) Studiamo dapprima alcune proprietà degli eventuali punti fissi di $\mathfrak{C}$.

E di immediata dimostrazione il seguente

Lemara 4.1. - Se $u \in W^{2,6}(D)$ verifier $u=\mathfrak{G} u$ allora $\grave{e} u_{0} \leqslant u \leqslant u^{0}$. In particolare $u \geqslant 0$.

LEMMa 4.2. $-\$ e u \in W^{2,6}(D)$ verifica $u=\mathfrak{G} u$ allora $u_{z} \geqslant 0$ in $\bar{D}$.

DiM. - Si supponga che l'aperto $\omega=\left\{(x, y, z) \in D: u_{z}(x, y, z)<0\right\}$ sia non vuoto. Allora in $\omega$ è strettamente crescente la funzione $z \rightarrow\left(\$ \gamma_{3} u\right)(x, y)-u(x, y, z)$ e dunque è non decrescente la funzione $z \rightarrow \Delta u(x, y, z)$, da cui $\Delta u_{z}=(\Delta u)_{z} \leqslant 0$ in $\omega$.

Siccome $u_{z}$ non può essere costante in $\omega$, tutti i punti di minimo per $u_{z}$ in $\bar{\omega}$ stanno su $\partial \omega$, ma non su $\left(\Gamma^{\prime} \cup \Gamma^{\prime \prime}\right) \cap \partial \omega$, in quanto con opportuno ribaltamento ci si riconduce alla situazione dei punti interni, nè su $D \cap \partial \omega$ ove $u_{z}=0$, nè su $\partial \omega \cap \bar{\Gamma}$ ove $u_{z}=g \geqslant 0$, e nemmeno su $\bar{\Gamma}_{0}$ ove $u_{z} \geqslant 0$ come conseguenza del lemma 4.1. Siamo dunque pervenuti ad un assurdo, da cui la tesi.

Lemma 4.3. - Se $u \in W^{2,6}(D)$ verifica $a t=6 u$ allora $\grave{e}$

$$
\left(\frac{\partial^{2}}{\partial x^{2}}+\frac{\partial^{2}}{\partial y^{2}}\right) \gamma_{3} u \geqslant 0 \quad \text { in } B_{3}
$$

Din. - Proviamo che $u(x, y, z) \leqslant\left(\mathrm{S} \gamma_{3} u\right)(x, y)$ in $\bar{D}_{3}$.

Per assurdo sia $\alpha=\max \left\{u(x, y, z)-\left(\delta \gamma_{3} u\right)(x, y):(x, y, z) \in \bar{D}_{3}\right\}>0$. Per il lemma 4.2, $\alpha$ deve essere assunto in almeno un punto di $\bar{\Gamma}_{3}$. Essendo $u=\gamma_{3} u=\delta \Gamma_{3} u s u \partial \Gamma_{3}$ (per definizione di $\$$ ), l'insieme $\omega=\left\{(x, y) \in B_{3}: u(x, y, Z(x, y))-\left(\$ \gamma_{3} u\right)(x, y)=\alpha\right\}$ risulta non vuoto. $\omega$ è ovviamente chiuso in $B_{3}$. Mostriamo che è aperto.

Sia $\left(x_{0}, y_{0}\right) \in \omega$; si ha: $u\left(\infty_{0}, y_{0}, Z\left(x_{0}, y_{0}\right)\right)>\left(\delta \gamma_{3} u\right)\left(x_{0}, y_{0}\right)$, e quindi $\Delta u=0$ in un intorno di $\left(x_{0}, y_{0}, Z\left(x_{0}, y_{0}\right)\right)$. D'altra parte è $\left(\delta \gamma_{3} u\right)\left(x_{0}, y_{0}\right)<u\left(x_{0}, y_{0}, Z\left(x_{0}, y_{0}\right)\right)=$ 
$=\left(\gamma_{3} u\right)\left(x_{0}, y_{0}\right)$ e dunque

$$
\Delta\left(\delta \gamma_{3} u\right)=\left(\frac{\partial^{2}}{\partial x^{2}}+\frac{\partial^{2}}{\partial y^{2}}\right)\left(\delta \gamma_{3} u\right)=0
$$

vicino a $\left(x_{0}, y_{0}, Z\left(x_{0}, y_{0}\right)\right)$.

Si è allora provato che la funzione $\delta(x, y, z)=u(x, y, z)-\left(\$ \gamma_{3} u\right)(x, y)$ è armonica nell'intorno di $\left(x_{0}, y_{0}, Z\left(x_{0}, y_{0}\right)\right)$ che risulta punto di massimo per $\delta$. Essendo $\delta_{z}\left(x_{0}, y_{0}, Z\left(x_{0}, y_{0}\right)\right)=0, \delta$ risulta costante in un intorno di $\left(x_{0}, y_{0}, Z\left(x_{0}, y_{0}\right)\right)$. In particolare risulta costantemente uguale ad $\alpha$ in un intorno di $\left(x_{0}, y_{0}\right)$ la funzione $u(x, y, Z(x, y))-\left(\delta \gamma_{3} u\right)(x, y)$. $\omega$ è dunque aperto.

Essendo $B_{3}$ connesso, deve essere $\omega=B_{3}$. Ma questo è assurdo in quanto risulterebbe $u(x, y, Z(x, y))-\left(\delta \gamma_{3} u\right)(x, y)=\alpha>0$ anche $s u \quad \partial B_{3}$, che contraddice la definizione di $\delta$. Dunque $u(x, y, z) \leqslant\left(\delta \gamma_{3} u\right)(x, y)$ in $\bar{D}_{3}$ : Segue in particolare $\gamma_{3} u \leqslant \delta \gamma_{3} u$ in $\bar{B}_{3}$ e dunque $\gamma_{3} u \equiv \mathrm{S}_{3} u$ in $B_{3}$, da cui la tesi, grazie alla definizione stessa di S. c.v.d.

2) Siamo ora in grado di dimostrare il:

TeOREMa 4.1. - $U$ è soluzione del problema 1.1 se e solo se $U \in W^{2,6}(D)$ e $U=\mathfrak{G} U$.

DIM. - Sia $U$ soluzione del problema 1.1. In particolare, grazie a (1.4) $\delta \gamma_{3} U$ è definita e ovviamente $U=6 U$. Per il teor. 2.3 è allora $U \in W^{2,6}(D)$.

Sia ora $U \in W^{2,6}(D)$ tale che $U=\mathscr{C} U$. Valgono allora (1.4), (1.7), mentre (1.5), (1.6) sono date dai lemmi 4.3 e 4.2 .

In particolare da $U=\mathcal{C} U$, segue $0 \leqslant-\Delta U \leqslant 1$ in $D$ e, con la definizione (1.8), $-\Delta U=1$ in $\Omega$.

D'altra parte è applicabile il corollario 3.1 con $w=U$ che, unito al lemma 3.3 e a (1.5), fornisce $\gamma_{3} U \in W_{\text {loc }}^{2, \infty}\left(B_{3}\right)$. $\mathrm{Ma}$ (1.6) assicura che $U \leqslant \gamma_{3} U$ in $D$ e quindi $\Delta U=\Delta \gamma_{3} U$ q.o. in $D-\Omega$.

$\Delta U \leqslant 0$ in $D$ e. (1.5) forniscono allora $\Delta U=0$ q.o. in $D-\Omega$, da cui (1.10).

Infine (1.9) segue dal fatto che, per il principio di Hopf, risulta $U_{z}>0$ su $\Gamma_{0}$.

Dunque $U$ risolve il problema 1.1. e.v.d.

Osservazione 4.1. - Il problema della ricerca dei punti fissi per $\mathcal{C}$ è effettivamente di natura quasi-variazionale $\left({ }^{16}\right)$ nel senso di [20] (per una situazione analoga v. [5]); tuttavia il metodo di [20] non è direttamente applicabile nel caso in questione.

\section{5. - Ricerea dei punti fissi di $\mathfrak{G}$.}

1) Con la notazione (3.15), poniamo:

$$
U_{0}=u_{0} ; \quad U^{0}=u^{0} ; \quad U_{n+1}=\mathscr{C} U_{n} ; \quad U^{n+1}=\mathscr{C} U^{n} ; \quad \forall n \geqslant 0 .
$$

${ }^{\left({ }^{16}\right)}$ Le disequazioni quasi-variazionali, introdotte dapprima in [10], sono state studiate suecessivamente ad es. in [13] e [20]. 


$$
U_{\infty}=\lim _{n \rightarrow \infty} U_{n} ; \quad U^{\infty}=\lim _{n \rightarrow \infty} U^{n} \quad \text { puntualmente in } \bar{D} .
$$

Sussiste il seguente:

TEOREMA 5.1. - Le funzioni $U_{\infty}$ e $U^{\infty}$ sono ben definite, appartengono a $W^{2,8}(D)$ e sono il minimo e il massimo dei punti fissi $d i \mathfrak{C}$.

Drm. - Ragioniamo su $U^{\infty}$. 亡̀ $W^{0} \in W^{2,6}(D) \cap V$ e $u_{0} \leqslant U^{0} \leqslant u^{0}$. Procedendo per induzione, se $n \geqslant 0$ e $U^{n} \in W^{2,6}(D)$ verifica $u_{0} \leqslant U^{n} \leqslant u^{0}$, il teor. 3.3 e il lemma 3.2 assicurano che $U^{n+1}=6 U^{n}$ è ben definita, appartiene a $W^{2,6}(D)$ e verifica $u_{0} \leqslant U^{n+1} \leqslant u^{0}$.

Dunque la successione $\left\{U^{n}\right\}$ è ben definita e limitata in $C^{0}(\bar{D})$. Ancora il lemma 2.6 assicura che, essendo $u_{0} \leqslant U^{0} \leqslant u^{0}$, risulta $U^{1} \leqslant U^{0}$. Ragionando per induzione, se $n \geqslant 0$ e $U^{n+1} \leqslant U^{n}$, per il lemma 3.1 si ha $U^{n+2} \leqslant U^{n+1}$.

Dunque la successione $\left\{U^{n}\right\}$ è anche monotona non decrescente e la funzione $U^{\infty}$ è ben definita.

Per definizione di $U^{n+1}$, si ha

$$
-\Delta U^{n+1}+\lambda_{0} U^{n+1} \in \lambda_{0} U^{n}+\chi_{1}+\chi_{2}+\chi_{3} H\left(\delta \gamma_{3} U^{n}-U^{n+1}\right) .
$$

Per quanto si è provato, risulta dunque che la successione $\left\{-\Delta U^{n}+\lambda_{0} U^{n}\right\}$ è limitata in $L^{\infty}(D)$.

Per il teorema 2.1, $\left\{U^{n}\right\}$ risulta limitata in $H^{1}(D)$ e in $C^{0, \alpha}(D)$, per opportuno $\alpha \in] 0,1$ [. Siano allora $\left\{U^{n_{k}}\right\}$ una sottosuccessione convergente debolmente in $H^{1}(D)$ e fortemente in $C^{0}(\bar{D})$ e $U^{*}$ il relativo limite: risulta a $U^{*}=U^{\infty}$ e "tutta" la suecessione $\left\{U^{n}\right\}$ converge a $U^{\infty}$ debolmente in $H^{1}(D)$ e fortemente in $C^{0}(\bar{D})$. In particolare $\left.U^{\infty}\right|_{\Gamma_{0}}=0$.

Ma anche la suecessione $\left\{\Delta U^{n}\right\}$ è limitata in $L^{\infty}(D)$; dunque $U_{z \mid \Gamma}^{\infty}=g$ e $\partial U^{\infty} /\left.\partial y\right|_{\Gamma^{*} \cup \Gamma^{*}}=0$ nel «senso delle tracce».

D'altra parte risulta

$$
-\Delta U^{\infty}=-\Delta U^{\infty}+\lambda_{0}\left(U^{\infty}-U^{\infty}\right)=\lim _{n \rightarrow \infty}\left[-\Delta U^{n+1}+\lambda_{0}\left(U^{n+1}-U^{n}\right)\right] \quad \text { in } \mathfrak{D}^{\prime}(D) .
$$

In particolare $-\Delta U^{\infty} \in L^{\infty}(D) \mathrm{e}-\Delta U^{\infty} \equiv 1$ in $D_{1} \cup D_{2}$.

Per il teor. 2.3 (applicato con $\lambda=0$ ) si ha allora $U^{\infty} \in W^{2,6}(D)$. Infine dalla convergenza uniforme di $U^{n}$ a $U^{\infty}$, grazie a (3.11), si deduce la convergenza uniforme di $\delta \gamma_{3} U^{n}$ a $\delta \gamma_{3} U^{\infty}$ e dunque è facile passare al limite nella disequazione di (3.2) (scritta con $u=U^{n+1}, w=U^{n}, f=\S \gamma_{3} U^{n}, \lambda=\lambda_{0}$ ) ed ottenere $U^{\infty}=\mathscr{C} U^{\infty}$.

Sia ora $U \in W^{2,6}(D)$ verificante $U=\mathscr{G} U$.

Per il lemma 4.1 è $U \leqslant U^{0}$. Ragionando per induzione, se $n \geqslant 0$ e $U \leqslant U_{n}$, si ha (grazie al lemma 3.1): $U=\mathfrak{G} U \leqslant \mathcal{C}^{n}=U^{n+1}$. Dunque $U \leqslant U^{n} \forall n$ e quindi $U \leqslant U^{\infty}$ in $\bar{D}$, il che prova che $U^{\infty}$ è il massimo dei punti fissi per $\mathfrak{G}$.

Analogamente si dimostra che $U_{\infty}$ è il minimo dei punti fissi per $\mathcal{C}$. c.v.d. 


\section{BIBLIOGRAFIA}

[1] S. AGMon - A. Douglis - L. NIRENBERg, Estimates near the boundary for solution of elliptic partial differential equations satisfying general boundary conditions, $I$, Comm. P. A. M., 12 (1959), pp. 623-727.

[2] C. BAIocch, Su un problema di frontiera libera connesso a questioni di idraulica, Ann. Mat. pura e appl., 92 (1972), pp. 107-127; C. R. Acad. Sc. Paris, 273 (1971), pp. $1215-17$.

[3] C. Baroccht, Problèmes à frontière libre en hydraulique, C. R. Acad. Se. Paris, 278 (1974), pp. 121-124.

[4] C. BAIOCCHI, Free boundary problems in the theory of fluid flow through porous media, Proc. of the International Congress of Mathematicians, Vancouver (1974).

[5] C. BAIоcchI, Studio di un problema quasi-variazionale connesso a problemi di frontiera libera, Boll. U.M.I., (4), 11, Suppl. fasc. 3 (1975), pp. 589-613.

[6] C. BAIоcCHI, Inéquations quasi-variationnelles dans les problèmes à frontière libre en hydrautique, Applications of Methods of Functional Analysis to Problems in Mechanies, Lecture Notes in Mathematies, 503, pp. 1-7, Springer (1976).

[7] C. BaIOCCMI - V. Commctoli - L. GuERRI - C. VoLPI, Free boundary problems in the theory of fluid flow through porous media: a numerical approach, Calcolo, 10 (1973), pp. $1-86$.

[8] C. Baiocchi - V. Comincioli - E. Magenes - G. A. Pozzi, Free boundary problems in the theory of fluid flow through porous media: existence and uniqueness theorems, Ann. Mat. pura e appl., (4), 96 (1973), pp. 1-82.

[9] J. BEar, Dynamics of fluids in porous media, Amer. Els. Publ., New York (1972).

[10] A. Bensoussay - J. L. Lions, Nouvelle formulation de problèmes de contrôle impulsionnel, et applications, C. R. Acad. Sc. Paris Sér. A-B, 276 (1973), pp. A 1189-92.

[11] M. CHIcco, Principio di massimo per soluzioni di problemi al contorno misti per equazioni ellittiche di tipo variazionale, Boll. U.M.I., 3 (1970), pp. 384-394.

[12] G. GrLARDI, Studio di una famiglia di disequazioni quasi-variazionali connessa con un problema di frontiera libera, Boll. U.M.I., (5), 13-B, N. 1 (1976), pp. 138-159.

[13] J. L. Jotx - U. Mosoo, Sur les inéquations quasi-variationnelles, C. R. Acad. Sc. Paris, A 279 (1974), pp. 499-502.

[14] J. L. LIONs - E. MAGENEs, Non homogenous boundary value problems and applications, vol. I: Grundlehren, B. 181, Springer, Berlin (1972).

[15] J. L. Lions - G. Stampacchia, Variaional inequalities, Comm. P.A. M., 20 (1967), pp. $493-519$.

[16] M. K. V. MURthy - G. Stampacchta, A variational inequality with mixed boundary conditions, Jsrael J. of Math., 13, no. 1-2 (1972), pp. 188-224.

[17] M. H. Protter - H. F. Wennberger, Maximum principle in differential equations, Prentice-Hall, New Jersey (1967).

[18] G. Stampacchi, Formes bilinéaires coercives sur les ensembles convexes, C. R. Acad. Sc., 258 (1964), pp. 1413-16.

[19] $G$. Stampacchia, On the filtration of a fluid through a porous medium with variable crosssection, Russian Math. Surveys, 29, 4 (1974), pp. 89-102; Uspekhi Mat. Nauk, 29, 4 (1974), pp. 89-101.

[20] L. TARTAR, Inéquations quasi-variationnelles abstraites, C, R, Acad. Sc. Paris, 278 (1974), pp. $1193-96$. 